\title{
Management of Facial Scars
}

\author{
Velupillai Ilankovan and Anna Sayan
}

\subsection{Introduction}

When the body sustains injury, the process of wound healing will commence. Scar formation is a part of wound healing. These are described as primary, secondary and tertiary intention processes [1]. In primary, there is close approximation of the wound edges, and in the secondary, the edges could not be approximated. The wound is left open, and the defect is slowly filled with connective tissue. In the tertiary, however, there is infection or contamination and there is a delay until the offending items are removed. This is followed by primary intention healing.

An ideal scar is a thin line or lines in parallel to the relaxed skin tension lines (RSTLs) described by Borges [2]. This should have a natural contour, colour and no distortion of the surrounding structures.

The scar particularly the facial is not aesthetically pleasing and can cause psychological distress to patients, resulting in poor body image, reduced self-esteem, generally loss of confidence and at times certain social stigma.

Prevention of an unsightly scar is primarily important requirement to any Surgeons but particularly important to Facial Plastics and Aesthetic Surgeons.

The origin of the word scar comes from Greek termed "iskharo" and in French "eschori" and interestingly was first used in the English language in the fourteenth century.

The primary aim in the management of scar is to produce an invisible line.

Less than ideal scars demonstrate features that are highlighted in Box 36.1.

Multiple causes can play a role in unsightly scar formation. Non-clear wound edges cannot give an aesthetically pleasing outcome. Nutritional status, co-morbidities such as diabetes, previously radiotherapy field and habits such as smoking have a detrimental effect in wound healing and thus

V. Ilankovan $(\varangle) \cdot$ A. Sayan

Oral and Maxillofacial Surgery, Poole Hospital NHS Foundation

Trust, Poole, UK

\section{Box 36.1 Characteristics of less than ideal scars}

- wide

- raised

- depressed

- pigmented

- hypo-pigmented

- and erythematous

- may transect natural creases, folds and junctions

imperfect scars. The most important factor is also the surgical competence of the operator.

\subsection{Pathophysiology}

The pathophysiology of wound healing and scar formation is very complicated [3].

The wound healing is characterised by four separate but overlapping phases (Box 36.2).

Haemostasis and inflammation occur during the first 4-6 days. During the first few minutes after surgery, there are vascular contraction and formation of fibrin clot. The ruptured cell membranes result in the release of inflammatory factors in order to cause vasoconstriction, which will last for 5-10 min, and then the vessel dilatation starts. This process can take up to $20 \mathrm{~min}$. The vessel dilatation is caused by histamine, which, in turns, allows inflammatory cells to arrive at the wound site. The platelets also adhere to the site of injury. Subsequently, the coagulation cascade will be activated. The structure of the clot will be a fibrin protein network filled with platelets and its contents. The main inflammatory mediators released by the platelets are the prostaglandins, leukotrienes, interleukins, growth factors and cytokines. During the inflammatory phase, there is also the arrival of neutrophils, macrophages and lymphocytes. The main function of the neutrophils is the removal 


\section{Box 36.2 Phases of wound healing}

- haemostasis

- inflammation

- proliferation

- remodelling

of the invading materials such as the microbes and products from the cell death. The macrophages are, however, responsible for inducing and clearing dying cells, which will result in improvement of inflammation. The apoptotic cells are cleaned by the macrophages, and the reparative stage begins. During this stage, there is migration of the epithelial cells usually after 48 hours and this process continues until the tenth day. Fibroblasts promote collagen synthesis. The epidermis is thickened in layers, and the collagen fibres promote dermal strength. The collagen deposition takes 2-3 weeks, and at this time, the remodelling phase begins. The collagen fibres will become rearranged during this period, and the cross-linked will be aligned along the tension lines. Largely, the maturation phase can take up to a year or longer depending on the type and site of wound. Therefore, the inflammatory process plays a major role in scar formation with the resultant fibroblast proliferation migration and differentiation.

The formal scar can be made better by using scar and scar reducing agents such as non-steroidal anti-inflammatory drugs (NSAIDs), minocycline and gene therapy. The main recognised scar reducing agents are transforming growth factor (TGF), TGF beta 3, COX-2 inhibitors and angiotensin receptor blockers [4]. The TGF beta modulating agents are still undergoing phase 2 trials in order to find out ideal scar modulating topical or intralesional agents.

Microfat grafting [5] is a technique with great potential in scar management where fat can be used intralesionally using a small $0.7 \mathrm{~mm}$ diameter cannula. It has been shown to be even more effective in placing fat in deep dermal layer of the skin using a 23-gauge needle in a treatment called sharp needle intradermal fat (SNIF) grafting. However, the work carried out by Tonnard et al. [6] in emulsifying the fat filtered until a liquid suspension was obtained, called Nanofat, which is injected using a 27-gauge needle, which conveys excellent outcome.

The ideal scar is an invisible line. There was increased interest in the recent past on intrauterine surgery to improve craniofacial clefts with some success and large failures. The extrauterine scars can present as stretched to depressed, hypertrophic or at times keloid. The stretched and depressed scars are as a result of inadequate primary intention management. The differences between a hypertrophic scar and keloid scar are highlighted in Box 36.3.

\section{Box 36.3 Hypertrophic scar vs Keloid}

Hypertrophic scar

do not grow beyond the

boundaries of the original

wound

occur when there are skin wounds of any aetiology Not genetic

Not related to any race

Equal sex predilection
Keloid grow horizontally and outgrowing wound boundaries can arise from small injuries such as piercing of ears genetic More in dark skinned More in females

\subsection{Scar Management}

\subsubsection{General Principles}

Scar management includes prevention, pre-injury treatments during the healing period and definitive treatment once the scar is established.

It is essential to take accurate history about any previous scar formation. If it is known, extra precautions should be taken, patients should be alerted and incisions along the susceptible sites should be omitted.

For example, when doing blepharoplasty with a history of previous hypertrophic scar, we should avoid a subciliary incision and the treatment should be carried out via a transconjunctival approach. Avoidance of treatment should be practised, for example, a patient who had keloid on previous ear-piercing episodes.

Limiting skin stretching during wound healing, facilitating appropriate wound resting and preventive exercises in certain sites such as chest and suprapubic areas are observed. Atkinson et al. [7] reported a randomised control trial of the effect of tape fixation and the prevention of hypertrophic scars post-caesarean section with significant scar reduction.

Therefore, scar reduction therapies are classified into:

- topical,

- intralesional,

- radiation and

- scar revision with Z plasty, local flaps and skin grafts.

The latest progress in scar management includes treatment with lasers to reduce angiogenesis, subcision and intra- 
dermal fillers. The emulsified fat (NanoFat) with stromal vascular fraction (SVF) and plasma rich protein (PRP) usage is the current trend in the non-surgical outcome of scar management.

\section{Box 36.4 Non-surgical remedies}

- Steroid

- $5 F U$

- Belonycin

- Lasers

- Dermabrasion

- Subcision

- Fillers

- Silicone gel sheets

- Pressure therapy

- Cryotherapy

- Radiation

- Emulsified fat for scar rejuvenation

\subsubsection{Non-surgical Remedies}

The non-surgical modalities of scar management are highlighted in Box 36.4, and the description of each is as follows:

\section{- Steroid}

Triamcinolone is the mostly used steroid for scars. The mode of action when used as an intralesional therapy is in inhibition of the inflammatory mediators, inhibition of fibroblast proliferation, collagen synthesis and inhibition of TGF_BETA 1 and BETA 2-and enhancement of collagen degradation and collagenase action in keratinocytes [8].

The published reports, however, give varying outcomes with dose-dependent side effects such as hypopigmentation, telangiectasia and dermal atrophy [9]. There is an encouraging outcome, however, in combination therapy when the dosage is reduced.

\section{- $5 F U$}

The suggested mode of action is an inhibition of fibroblast proliferation and TGF-BETA 1-induced collagen synthesis [10]. Anecdotal results are positive; however, trials lack adequate controls. Nonetheless, the effect is encouraging in combination therapy with steroid and pulse dye lasers. Much work is required in controlled studies.

\section{- Bleomycin}

It is an antibiotic and has been used intralesionally in vascular malformation with good outcome [11].

The mode of action is in reduction of collagen synthesis and increased destruction of collagen by inhibiting Lysyl Oxidase such as TGF-B1 [12]. Much work is needed in its definitive usage.

\section{- Lasers}

Lasers target three chromophores such as water, melanin and haemoglobin. Laser-induced tissue hypoxia results in the breakdown of disulphide bonds of the collagen fibrils. Pulse Dye Laser (PDL), KTP laser and Nd YAG lasers have affinity to haemoglobin, leading to collagen necrosis, and results in reduction of proliferation of fibroblast and deposition of collagen type 3. Ablative lasers remove the scar layer by layer with minimal thermal injury [13].

\section{- Dermabrasion}

This technique is still used in treating irregular scars such as ice pick scars of acne. The aim is to abrade up to the reticular dermis, allowing epithelialisation to take place. This treatment causes erythema for a long time, and the symmetrical outcome is not guaranteed.

Microdermabrasion is an advancement where there is high-speed pressurised aspiration-compression system. The benefit is that it minimises deep thermal injury [14].

Carbon Dioxide $\left(\mathrm{CO}_{2}\right)$ and Erbium laser resurfacing supersedes microdermabrasion with minimal thermal damage. Laser resurfacing acts in a different way compared to the vascular lasers. The vascular laser action is as a nonablative principle, whereas $\mathrm{CO}_{2}$ and Erbium cause ablation.

Vascular lasers decrease the prominence by destroying the blood vessels and stimulating collagen remodelling, thus softening the scar, whereas the ablative lasers such as $\mathrm{CO}_{2}$ and Erbium ablate or vaporise the excessive tissues. $\mathrm{CO}_{2}$ can induce more collagen remodellation and promote wound contraction [15].

The vascular lasers are used in hypertrophic and keloid scars. $\mathrm{CO}_{2}$ and Erbium are useful in stretched, asymmetric and irregular scars. There is a restriction as it is not effective in all skin types as resurfacing can result in excessive pigmentation in dark, Asian and African skins.

\section{- Subcision}

It is a technique where depressed scars are elevated, and the controlled trauma can aid in formation of new connective 
tissue. This is carried out by custom-made subcision knives or 20 gauge needles. It can be combined with autologous fat injection to the subcised areas filling in the space and simultaneous laser resurfacing [16].

\section{- Fillers}

Synthetic Hyaluronic acid, calcium hydroxylapatite and bovine collagen are used in aesthetic surgery for voluminisation. They can be used in treating depressed scars. However, these agents cannot be used in a larger quantity as they will cause subcutaneous nodules and inflammation, and in bovine collagen, there is $3 \%$ incidence of allergic history. There is also a need for repeat injection. Autologous fat has taken over as a material of choice for filling in scar management.

\section{- Silicone gel sheets}

These sheets are used prophylactically in known patients for bad scars as well as therapeutically. However, the outcome for hypertrophic scars is still questionable and inconclusive.

It appears that controlled studies have shown significant beneficial outcome in scar volume when the silicone sheets are used or not used in mirror image wounds. A Cochrane review of 13 trials involving 559 patients, however, con- cludes weak evidence in preventing hypertrophic scars in susceptible patients [17].

The mechanism of action is in producing hydration to the scars by occlusion.

\section{- Pressure therapy}

It is a perceived therapy that there is positive evidence in the literature. The largest randomised trial showed no significant difference in scar reduction with pressure therapy when compared to the controlled group [18].

\section{- Cryotherapy}

A large uncontrolled study of 135 patients with 166 keloids demonstrated $79.5 \%$ response rate with $80 \%$ reduction in scar volume. However, the results are not reproducible. This treatment causes atrophic, depressed scars with residual hypopigmentation [19].

Therefore, the usage of cryotherapy particularly on the face should be discouraged as a modality.

\section{- Radiation}

It is an effective adjuvant to surgical excision particularly to keloid. The effect is caused by inhibition of proliferation
Fig. 36.1 (a) Keloid Scar right ear lobule. (b) Postoperative photograph following scar excision and one dose of radiotherapy
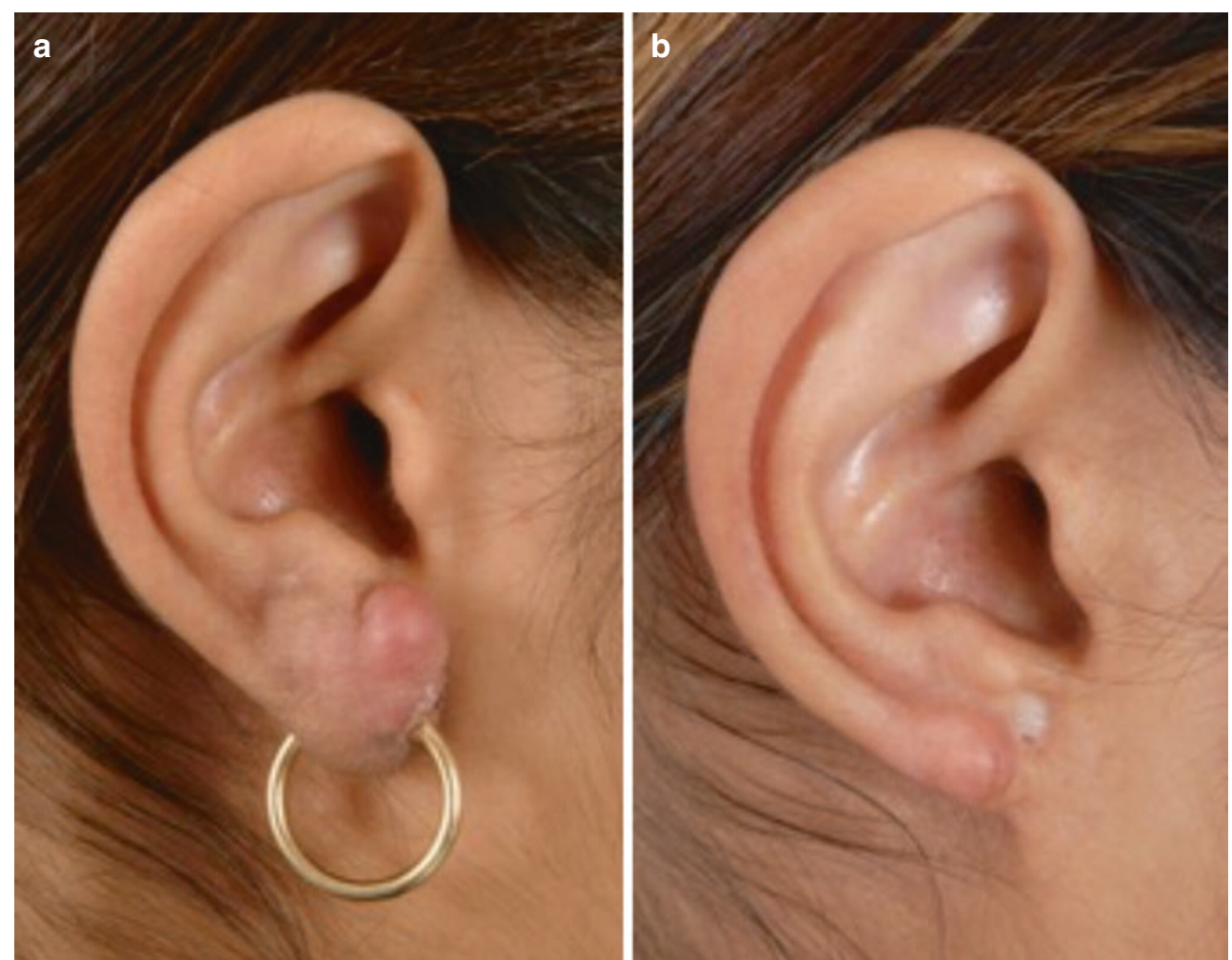

(CAssociation of Oral and Maxillofacial Surgeons of India 

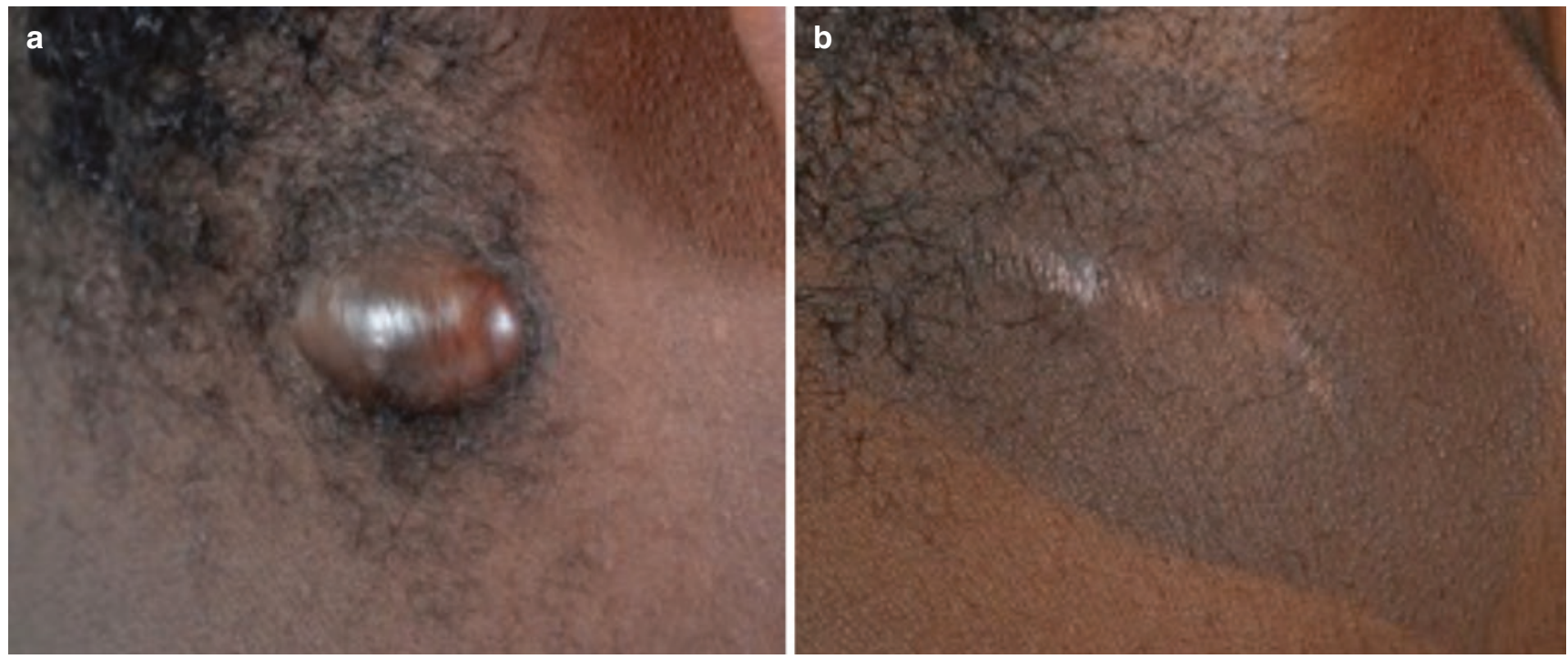

(c)Association of Oral and Maxillofacial Surgeons of India

Fig. 36.2 (a) Keloid Scar post-auricular region. (b) Post-operative photograph following scar excision and one dose of radiotherapy

of fibroblasts and neo-vascular blood formation, resulting in decreased collagen production [20].

The highest cure rate is described in a single dose within 24 hours after surgical excision; however, further studies are needed to evaluate the dosage and to obtain optimum results (Figs. 36.1 and 36.2).

\section{- Emulsified fat for scar rejuvenation}

The fat tissue is accepted not only as a simple layer of insulation and as a storage for energy but also as a complex endocrine organ, which is intrinsically involved in metabolism and immunomodulatory activities and can provide multi-source resources for stem cells and other undifferentiated cell population, which is essential for regeneration.

In addition to the adipocytes in the fat tissue, there are other cell groups that are functionally very important such as stromal vascular fraction (SVF) and cell-assisted lipotransfer (CAL). The SVF of adipose tissue is a rich source of epithelial progenitor cells, T cells, B cells and adipose tissue macrophages [21]. Separation of SVF is a time-consuming and expensive process requiring collagenase treatment and centrifugation. The recent clinical results obtained with Nanofat grafting where the cells are emulsified show greater value than the components of SVF used separately.

In Nanografting, a 23 gauge needle is used as a sharp needle for subdermal fat injection technique (SNIF) followed by a 27 gauge needle to superficial layers. In order to do this, the fat should be emulsified and takes the name, thus, Nanofat [6].

This now emerges to the forefront in regenerative medicine due to its ability to differentiate into a variety of different cells of cell lineage and the anti-apoptotic, antiinflammatory, pro-angiogenic immunomodulators and antiscarring properties.

Nanofat has shown to improve the aesthetics of hypertrophic and keloid scars by improving the texture of the scars in addition to improving the symptoms of pain and itching [22].

Fat is used in voluminisation initially as foundation micrograft just above the periosteum. The second layer is subdermal graft followed by subcision and lastly Nanofat injection using SNIF technique with 23-gauge needle followed by superficial needle injection using 27 -gauge needle. This process is to treat depressed scars. Intralesional Nanofat injection improves hypertrophic and keloid scars. This can be combined with other modalities such as laser resurfacing.

\subsection{Surgical Scar Management}

\subsubsection{Basic Principles}

As described above, an ideal scar should be an imperceptible line parallel to RSTL. The only exception is scar along the lower lid due to the possibility of ectropion, if incisions are made in parallel to RSTL.

The aim of scar revision is to re-orientate the scar to follow the RSTLs and correction of adjacent structure distortion.

Patient understanding, nutrition, expectation and the psychological stability are paramount factors to implement preoperatively prior to any scar revision exercises particularly in burn scars. The timing of the revision is also important as the remodelling may take up to 12 months. 
Nutritional status and the medical history are important pre-operative considerations. It has been described with certain evidence that the use of vitamins $\mathrm{A}, \mathrm{C}$ and $\mathrm{E}$ and zinc have beneficial effects and, at the same time, some herbal supplements such as arnica, garlic, ginseng and saw palmetto can impair wound healing [22]. Systemic conditions such as diabetes and immunosuppression can adversely affect wound healing. Smoking has proven to cause perilous effect on healing and wound repair.

Vitamin E refers to a group of ten lipid soluble compounds that include both Tocotrienols and Tocopherols as a fat soluble antioxidant; it stops the production of reactive oxygen species formed when fat undergoes oxidation [23]. It has been identified that alpha-tocopherol, the most biologically active form of vitamin $\mathrm{E}$, has anti-inflammatory effects by decreasing plasma C-reactive protein (CRP) levels and release of pro-inflammatory cytokines. The CRP, a downstream marker of inflammation in addition to being a risk marker for cardiovascular disease, could contribute to atherosclerosis. Alpha-Tocopherol has been shown to decrease CRP level in patients and has a positive effect in scar reduction. Topical vitamin $\mathrm{E}$ application has been practised in managing scar 10 days post-suture removal. Anecdotally, in the last 25 years, good scar outcome has been observed.

\subsubsection{Surgical Technique}

In most circumstances, incisions should be made parallel to the skin surface. A number 11 or 15 blade is ideal. Tissue handling must be atraumatic. The skin flaps should be held with non-toothed forceps, and the tooth forceps should only be used to handle tissues subdermally. Undermining and constant hydration of edges are essential. In stretched scars, preservation of the dermis by de-epithelialisation will provide extra support in correcting the depression. Haemostasis should be achieved using a bipolar diathermy again minimising thermal injury to the surrounding areas.

Suturing is done in layers. In areas where supportive tension is required, resorbable round body needle sutures are useful. Depending on the site and the extent of the scar, short- or long-term resorbable sutures could be selected. With deep suturing as much as possible, the knot should be buried with skin approximation to achieve 'eversion'. Simple-interrupted sutures are preferable in a majority of areas; however, subcutaneous running sutures could be used where only gentle approximation is needed.

\section{Z plasty (Fig. 36.3)}

This is a common technique used in scar revision to either change the direction of the scar or lengthen the scar. Contracted scars can be corrected by plating multiple simultaneous $\mathrm{Z}$ plasties.

The $\mathrm{Z}$ plasty is created by using the original scar as a central portion, and triangular flaps are raised. Multiple variations are in $\mathrm{Z}$ plasty. In the classical Z plasty, all the limbs are of equal length and the angle between the flaps is $60^{\circ}$. The scar will lengthen by $75 \%$. A $30^{\circ}$ angle will lengthen by $25 \%$, and $45^{\circ}$ will lengthen by $50 \%$. Multiple $\mathrm{Z}$ plasties are useful in long scars, which can be divided into separate segments, and the angles are contoured or modified depending on the sites.

\section{W plasty (Fig. 36.4)}

This technique is used to irregularise scars so that dimensions of the scar are not perceived by the naked eye. A series of consecutive triangles are made. It is mirror image on the opposite side. The arm length should be around $5 \mathrm{~mm}$, and the angle in-between the flap should be maximum $90^{\circ}$. One side of the triangle should be in parallel to our RSTL. The
Fig. 36.3 Z plasty (a) scar revision. (b) triangular flaps raised. (c) interposition of triangular flaps
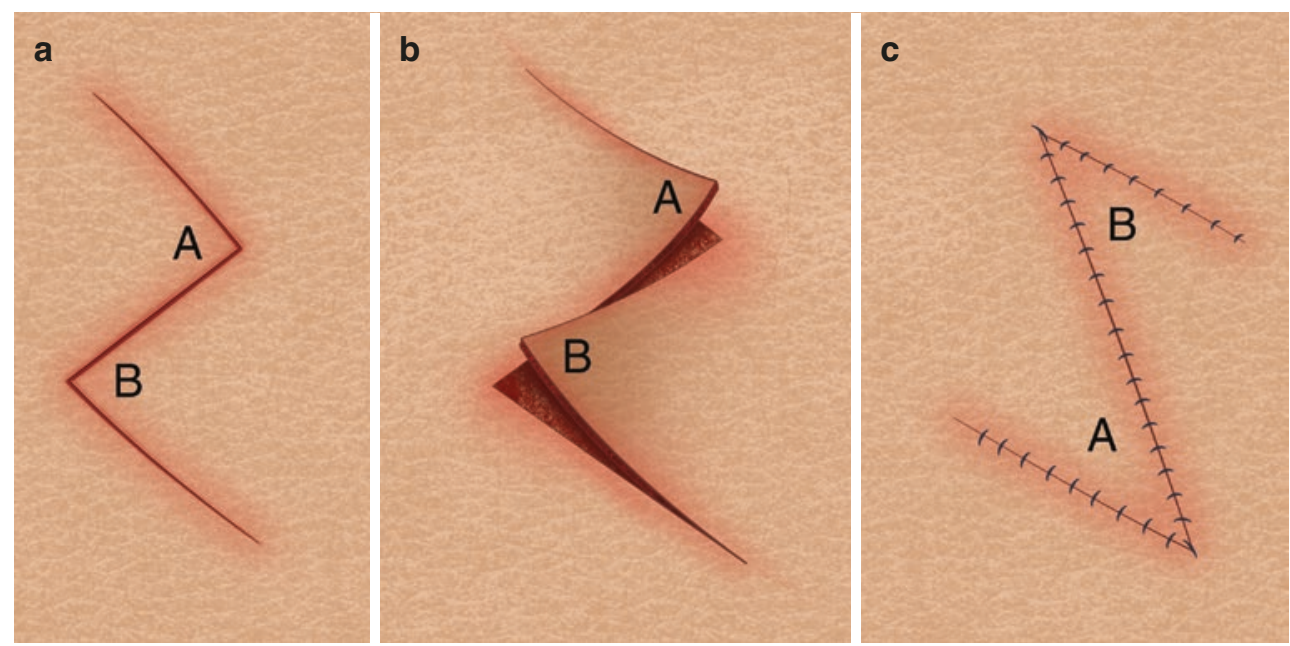

(CAssociation of Oral and Maxillofacial Surgeons of India 


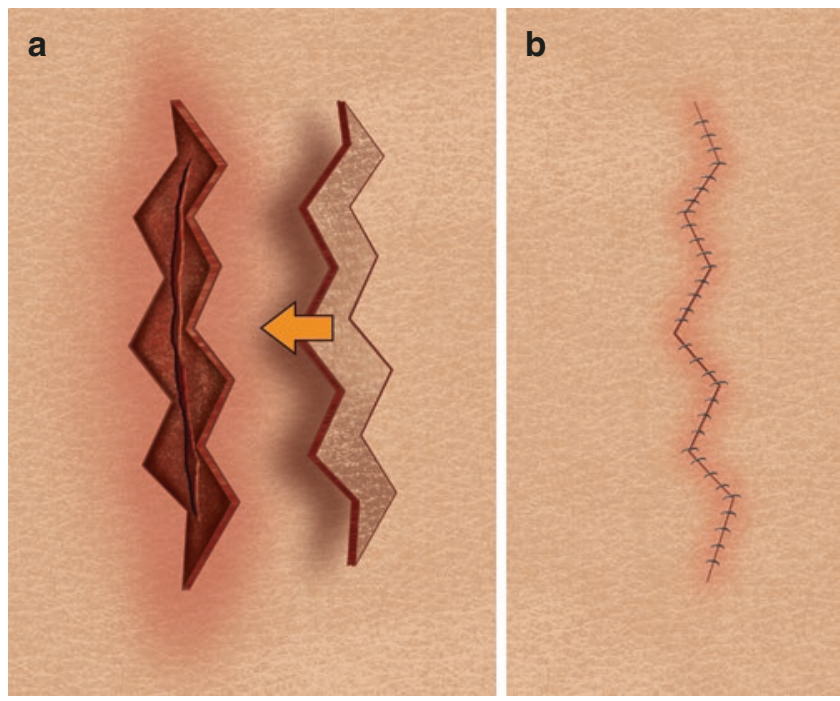

CAssociation of Oral and Maxillofacial Surgeons of India

Fig. 36.4 W plasty- (a) scar revision. (b) post-operative triangle are excised, undermined and closed with interdigitation. This technique is very useful in correcting unsightly hairline scars such as revision of face lift.

\section{Geometric broken line closure (GBLC)}

This is another technique to minimise the perception of a long scar. Here, random geometric designs are planned with corresponding mirror images. The length of the limbs should be $3-6 \mathrm{~mm}$ and at the angle near to $90^{\circ}$, and a W plasty could be done at the end of the design.

\section{4. $V$ - $Y$ and $Y$-V advancement}

V-Y scar correction (Fig. 36.5a-c) is utilised when there is an indication on scar lengthening in contracted scars with 'trap door deformity'. In contrast, Y-V technique is utilised where the scar is shortened, removing the ' $\mathrm{Y}$ 'extension (Fig. 36.6a-c).
Fig. 36.5 V-Y scar lengthening correction. (a) $\mathrm{V}$ incision. (b) Triangular flaps raised. (c) Y closure

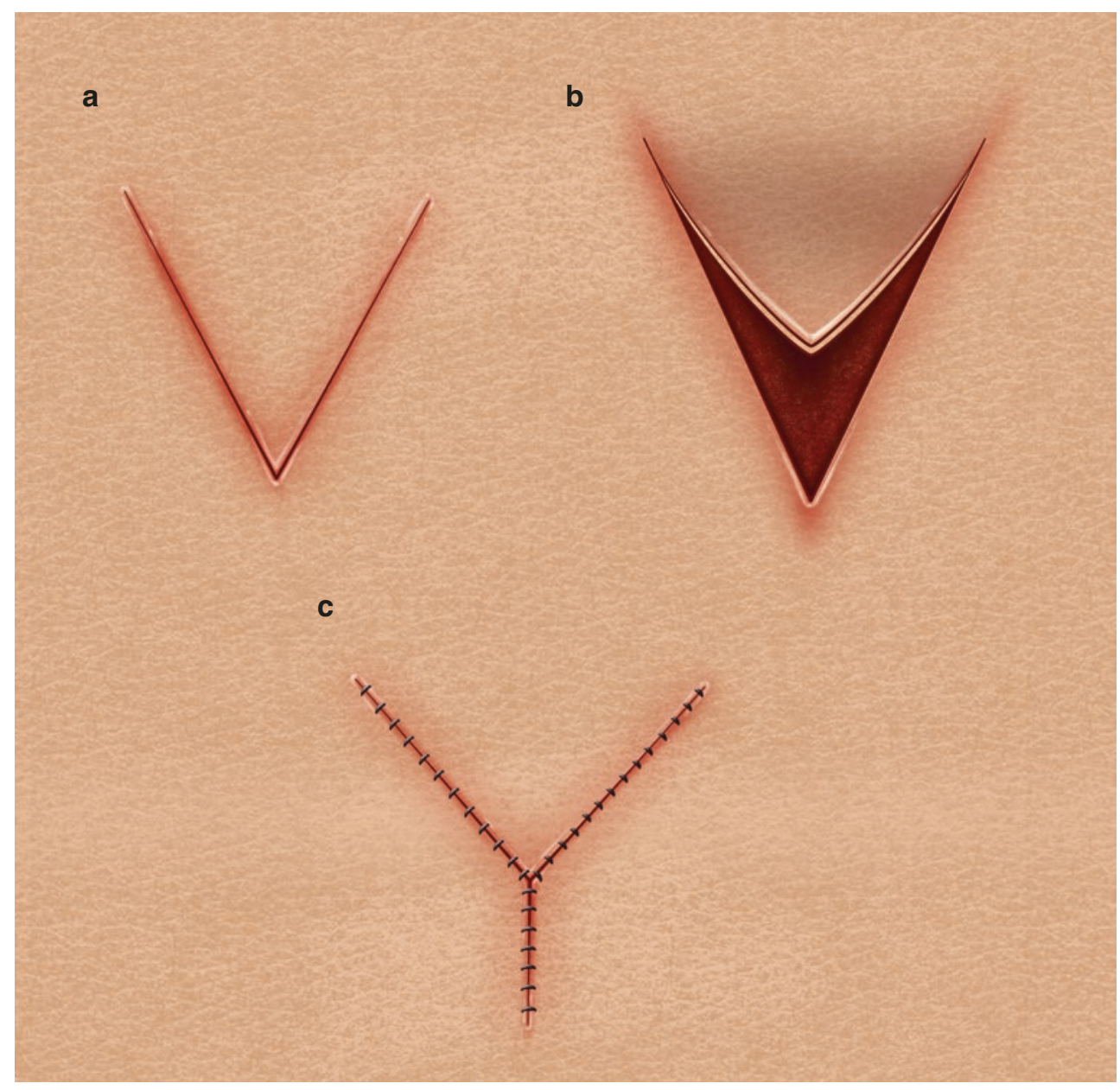

(CAssociation of Oral and Maxillofacial Surgeons of India 
Fig. 36.6 Y-V scar shortening correction. (a) $\mathrm{Y}$ incision. (b) Triangular flaps raised. (c) V closure

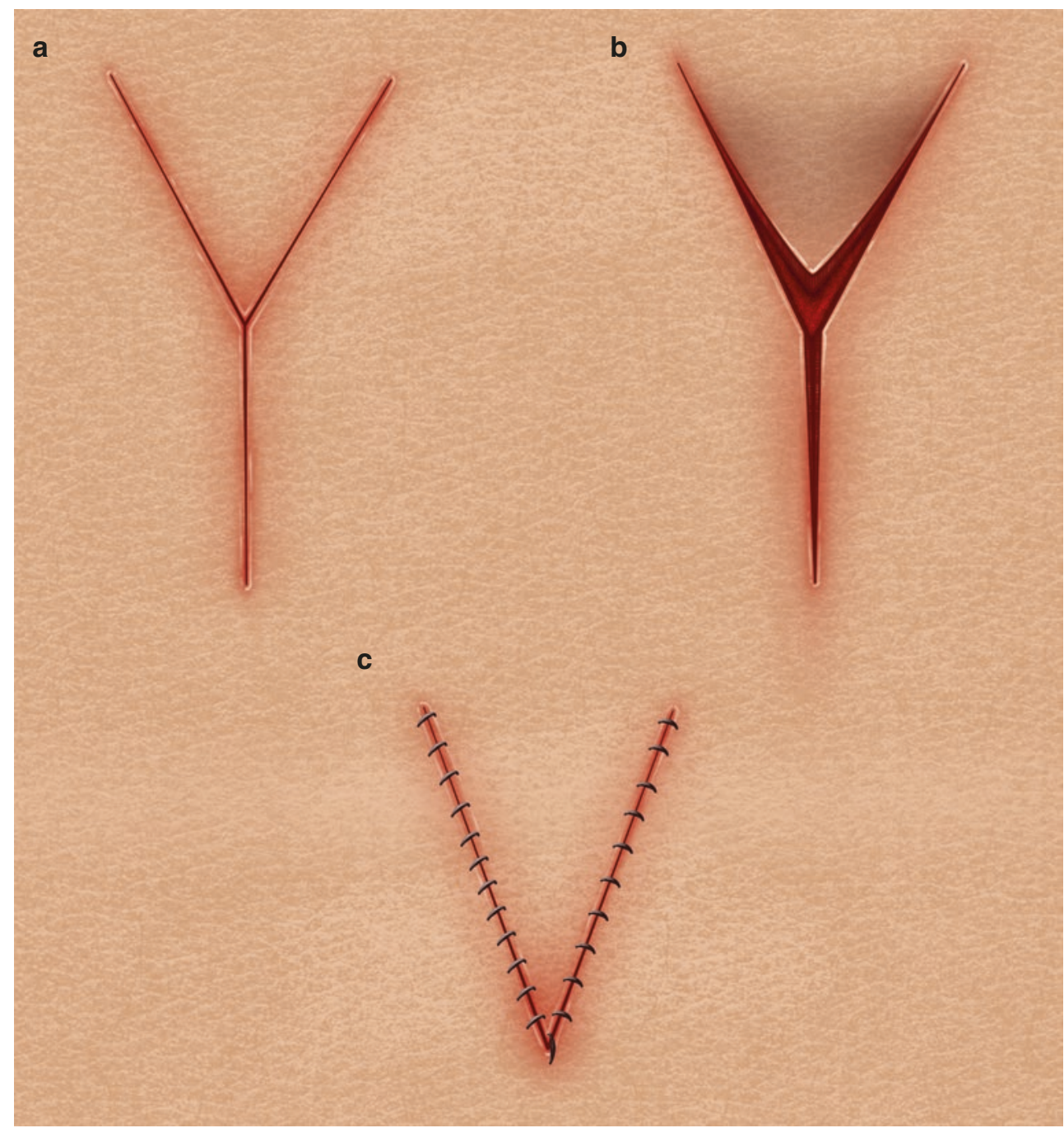

CAssociation of Oral and Maxillofacial Surgeons of India
Flaps can be used to revise large scars. "Doggy ear" and "trap door deformities"could be improved by excising the excess. For passive scar settlement, selective bulking and simultaneous multiple ' $\mathrm{Z}$ ' and ' $\mathrm{W}$ ' plasties are useful adjuncts.

\section{Clinical tip}

When repairing wounds in patients who are prone to develop hypertrophic scars, deep sutures should be placed using round bodied needles. The suture removal is delayed by an extra 3-4 days. Applying steri strips after removal of the sutures adds extra support, and this should be incorporated to achieve the best result.

Prophylactic intralesional steroids in patients prone to hypertrophic scars should be considered. Obliteration of the dead space by appropriate sutures or pressure dressings with or without drains should be considered to prevent haema- toma formation. If not, haematoma on its own will spoil the good work of scar revision.

\subsection{Future}

Extrauterine scarless surgery is not possible. Therefore, careful patient factor and scar characteristics should be studied to prevent unsightly scar formation and to correct perceptible scars.

General health and optimising comorbidities are important requirements to achieve good scars. Both non-surgical and surgical options should be available in the armamentarium to the Surgeon.

Nanofat has very positive potential in scar management. The research on gene therapy using adenovirus as a mediator to deliver dermal fibroblast is encouraging [24]. 


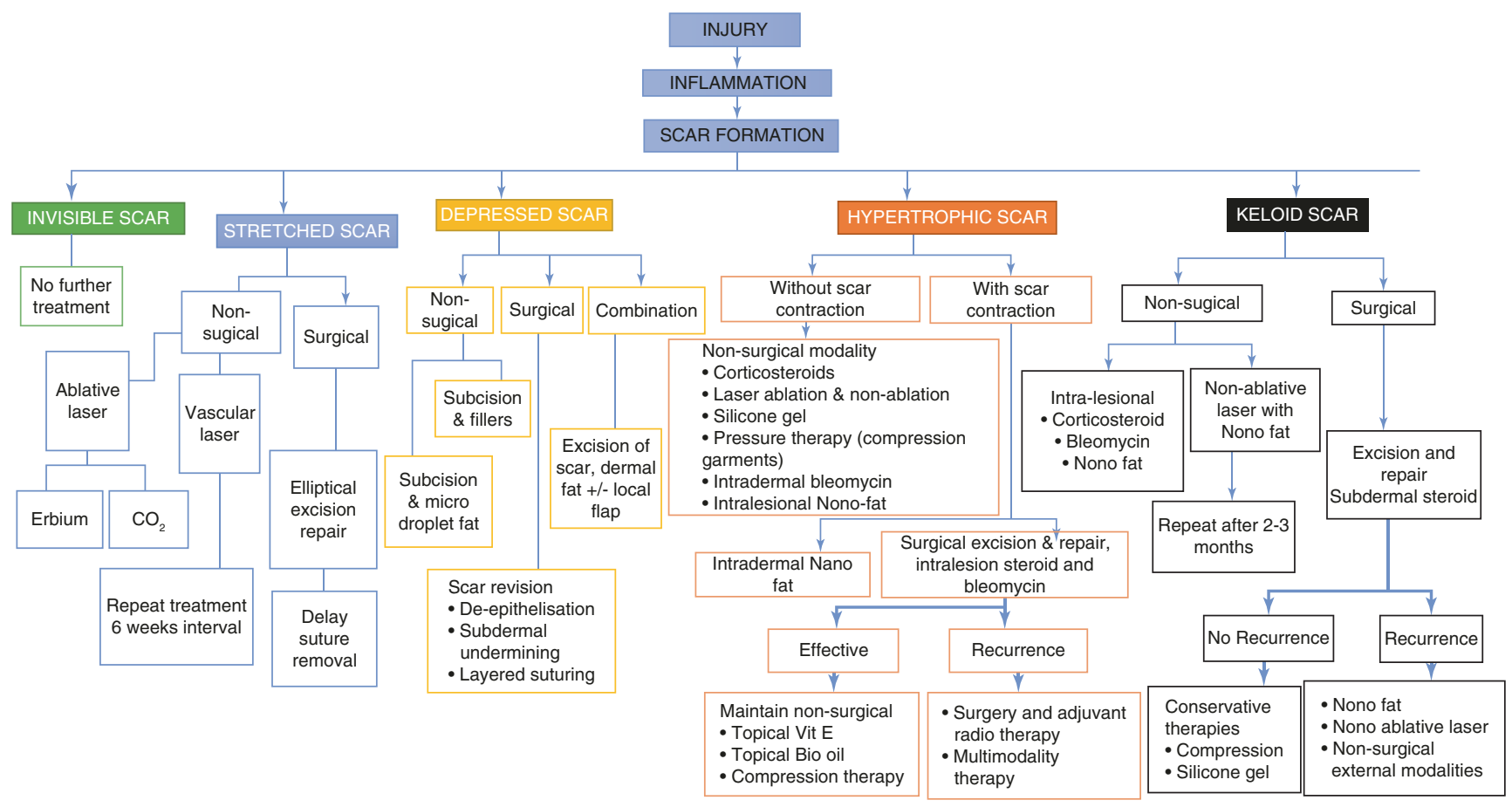

(C)Association of Oral and Maxillofacial Surgeons of India

Fig. 36.7 Algorithm for scar management

Although inflammatory response is traditionally believed to be a key event for wound healing in adult skin, studies of foetal wound healing suggest that a high level of inflammation might prevent good scar formation rather than enhancing the wound healing process. Therefore, further studies are needed to confirm if really inflammation is necessary for wound healing in adult skin. However, at present, we need to follow the inflammatory process and available evidence of therapeutic modulators of scar formation.

Furthermore, it is paramount to consider that there are clinical, histological, biochemical and molecular differences between hypertrophic and keloid scars. There is marked difference in the proliferative and apoptotic cell deaths between the scars, resulting in variable response of different treatments. This should be taken into account in treatment planning process.

It is also essential to avoid excessive movements that can stretch the wound and for avoidance of the scar to direct mechanical forces such as friction and scratching and particularly earlobe wounds, to minimise the contact with pillows and wound hygiene.

Not least the patient's involvement plays a vital role in the management of scars. Variable scales in the literature exist for reliable and reproducible scar management, for both the observer, i.e. surgeon, and the patient. The observer opinion is always influenced by vascularisation, thickness and pigmentation. The patient opinion, on the other hand, is influenced by action of itching sensation and thickness of the scar. Out of the available assessment scales, the patient and observer scar management described by Draaigers L et al. is an informative tool in documentation of the progress in the management of scar, which should be addressed [25].

In summary, a surgeon should have an algorithm to follow on how to manage scars in normal patients as well as patients who are prone for hypertrophic and keloid scars (Fig. 36.7).

\subsection{Conclusion}

Scar formation is a part of wound healing process. Facial scars can be aesthetically detrimental and affect social stigma. As facial surgeons, we need to achieve ideal scars with natural contour, colour and limited distortion. It is paramount to remember that scar management includes prevention, treatments during the healing period and definitive management once the scar has been established. We have, in this chapter, included all the different available treatments separating them into non-surgical remedies and surgical techniques. The algorithm, in this chapter, allows a very easy review process for surgeons who manage stretched, 
Fig. 36.8 (a) Pre-operative. (b) Post-"microfat grafting" and laser resurfacing
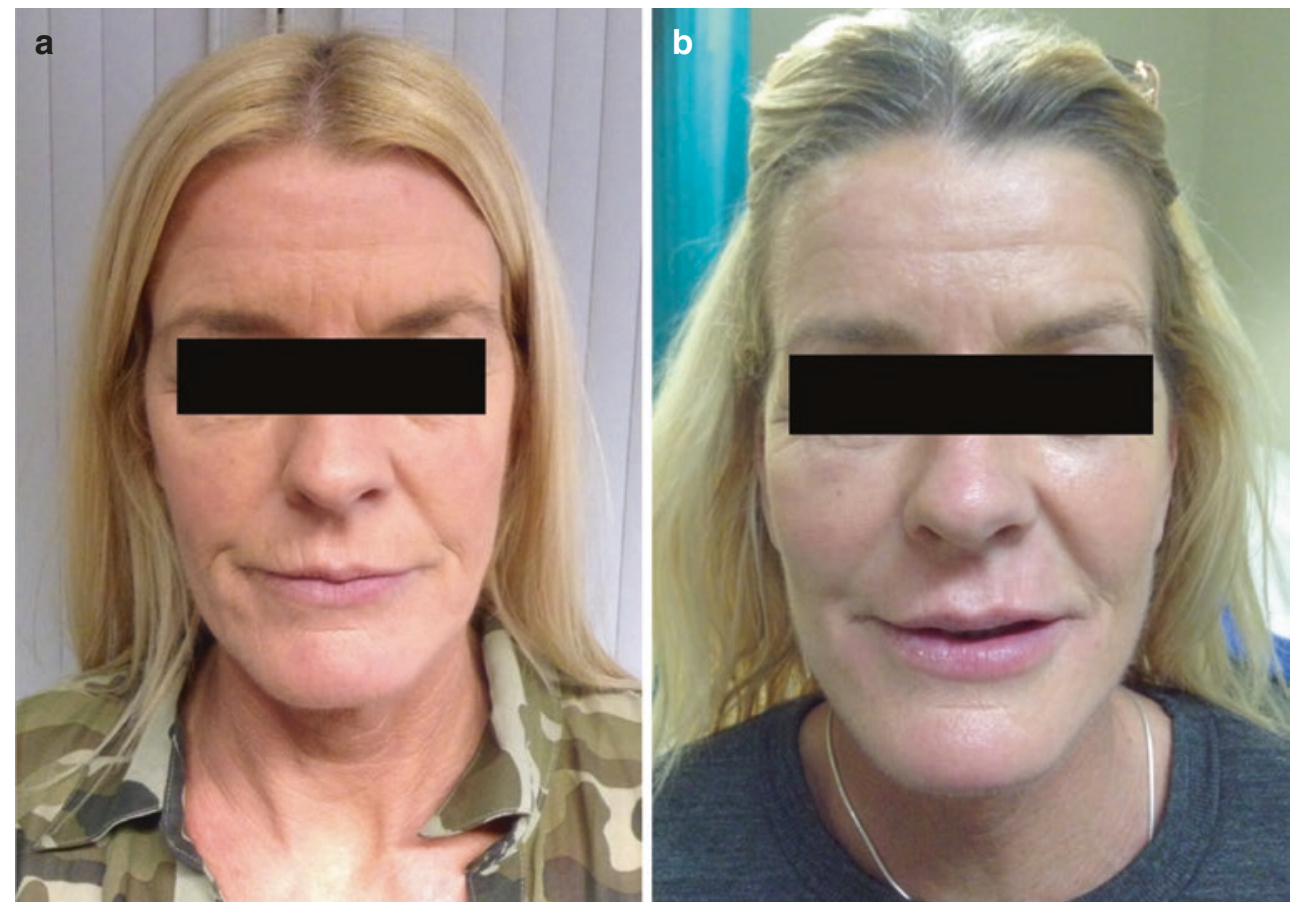

(CAssociation of Oral and Maxillofacial Surgeons of India depressed, hypertrophic and keloid scars.DisclosureAuthors have no financial conflicts to disclose.

\subsection{Case Scenarios}

\section{Case Scenario 1 (Fig. 36.1)}

A 27-year-old female patient of Asian ethnicity had an extra ear piercing to the right earlobe. Within 4 months, she started to develop a very painful hard lump at the pierced site. Within the following 6 weeks, it developed into an irregular mass involving the whole of the earlobe.

Her medical history was unremarkable, and she was not on any medication.

On examination (Fig. 36.1a), there was an unsightly thick lumpy scar along the right earlobe. A diagnosis of keloid was made.

Under a local anaesthetic, the keloid scar was excised and the earlobe was reconstructed. The next post-operative day she underwent single dose of radiotherapy (10 Gy) as an outpatient.

On review, 6 months post-treatment (Fig. 36.1b), she had no recurrence and on palpation, the skin was soft and non-tender.

\section{Case Scenario 2 (Fig. 36.8)}

A 48-year-old Caucasian patient who had a considerable amount of sun exposure presented with an unhappy peri-oral aesthetics (Fig. 36.8a).

Her medical history was unremarkable, and she was not on any medication.

In one examination, she had loss of volume along the peri-oral site mainly along the upper lip. The vermillion border had lost its definition. She had multiple small vertical subdermal lines, which were crossing the vermillion border. She also had epidermal thinning.

A diagnosis of aging of the peri-oral area, particularly upper lip, was made. She, in fact, had epidermal thinning, subdermal scaring and loss of inter- and intramuscular fat.

She underwent "microfat" grafting to restore the volume, subdermal "emulsified fat" to restore the subdermal structure and an "erbium laser resurfacing" (fluence $500 \mathrm{~mJ}$, mode: SP, spot $3 \mathrm{~mm}$, frequency $20 \mathrm{~Hz}$ ).

The post-operative appearance 4 months later (Fig. 36.8b) showed excellent volume restoration, much improvement in the subdermal scaring, better vermillion definition and a new regenerative skin.

This combination technique is very useful in burn scar management. 


\section{References}

1. Beldon P. Basic science of wound healing. Sci Direct. 2010;28:409-12.

2. Borges AF. Relaxed skin tension lines. Dermatol Clin. 1989;7:169-77.

3. Broughton G, Janis JE, Attinger CE. The basic science of wound healing. Plast Reconstr Surg. 2006;117:12S-34S.

4. Reish RG, Ericksson E. Scars: A review of emerging and currently available therapies. Plast Reconstr Surg. 2008;122:1068-78.

5. Collet C, Laversanne S, Haen P, et al. Micro-re-injection of autologous fat. Rev Stomatol Chir Maxillofac Chir Orale. 2013;114:381-6.

6. Tonnard P, Verpaele A, Peeters G, Hamdi M, Cornelissen M, Declercq H. Nanofat grafting: basic research and clinical applications. Plast Reconstr Surg. 2013;132:1017-26.

7. Atkinson JA, McKenna KT, Barnett AG, et al. A randomised controlled trial to determine the efficacy of paper tape in preventing hypertrophic scar formation in surgical incisions that traverse Langer's skin tension lines. Plast Reconstr Surg. 2005;116:1648-56.

8. Roques $\mathrm{C}$, Teot L. The use of corticosteroids to treat keloids. A review. Int J Lower Extremity Wounds. 2008.

9. Niessen FB, Spauwen PH. On the nature or hypertrophic scars and keloids: a review. Plast Reconstr Surg. 1999;104:1435-58.

10. Fitzpatrick RG. Treatment of inflamed hypertrophic scars using intralesional 5-FU. Dermatol Surg. 1999;25:224.

11. Sindel A, Sayan A, Ilankovan V, et al. Percutaneous treatment of orofacial vascular malformations. Br J Oral Maxillofac Surg. 2018;56:206-11.

12. Crooke ST, Bradner WT. Bleomycin, a review. J Med. 1976;7:333-428.

13. Lupton JR, Alster TS. Laser scar revision. Dermatologic Clin. 2002;20:55-65.

14. Tsai RY, Wang CN, Chan HL. Aluminium oxide crystal microdermabrasion. A new technique for treating facial scarring. Dermatol Surg. 1995;21:539-42.

15. Bowes LE, Nouri K, Bernam B, et al. Treatment of pigmented hypertrophic scars with the $585 \mathrm{~nm}$ Pulsed Dye Laser and the
$532 \mathrm{~nm}$ Frequency Doubled Nd: YAG laser in the Q-Switched and variable pulse modes: a comparative study. Dermatol Surg. 2002;28:714-9.

16. Orientreich DS, Orientreich N. Subcutaneous incisionless (subcision)surgery for the correction of depressed scars and wrinkles. Dermatol Surg. 1995;21:543-9.

17. Neissen FB, Spauwen PH, Robinson PH, Fedler V, Kon M. The use of silicone occlusive sheeting (Sil-K) and silicone occlusive gel (Epiderm) in the prevention of hypertrophic scar formation. Plast Reconstr Surg. 1998;102:1962-98.

18. Chnag P, Laubenthal KN, Lewos R, et al. Prospective, randomized study of the efficacy of pressure garment therapy in patients with burns. J Burn Care Rehabil. 1995;16:473.

19. Rusciani L, Paradisi A, Alfano C, et al. Cryotherapy in the treatment of keloids. J Drugs Dermatol. 2006;5:591.

20. Ragoowansi R, Cornes PG, Moss AL, et al. Treatment of keloids by surgical excision and immediate postoperative single-fraction radiotherapy. Plast Reconstr Surg. 2003;111:1853.

21. Zhu X, Du J, Liu G. The comparison of multilineage differentiation of bone marrow and adipose-derived mesenchymal stem cells. Clin Lab. 2012;58:897-903.

22. Broughton G, Crosby MA, Coleman J, et al. Use of herbal supplements and vitamins in plastic surgery: a practical review. Plast and Reconst Surgery. 2007;119:48-66.

23. Devaraj S, Leonard S, Traber M, Jialal I. Gamma-tocopherol supplementation along and in combination with alpha-tocopherol alters biomarksr of oxidative stress and inflammation in subject with metabolic syndrome. Free Radic Biol Med. 2008;44:1203-8.

24. Liu W, Chua C, Wu X, Want D, Ying D, Cui L. Inhibiting scar formation in rat wounds by Adenovirus-Mediated overexpression of trunated TGF-ß Receptor II. PRS. 2005;115:860-70.

25. Draaijers LJ, Templeman FR, Botman YA, Tuinebreijer WE, Middelkoop E, Kreis RW. The patient and observer scar assessment scale: a reliable and feasible tool for scar evaluation. PRS. 2004;113:1960-5.

Open Access This chapter is licensed under the terms of the Creative Commons Attribution 4.0 International License (http://creativecommons. org/licenses/by/4.0/), which permits use, sharing, adaptation, distribution and reproduction in any medium or format, as long as you give appropriate credit to the original author(s) and the source, provide a link to the Creative Commons license and indicate if changes were made.

The images or other third party material in this chapter are included in the chapter's Creative Commons license, unless indicated otherwise in a credit line to the material. If material is not included in the chapter's Creative Commons license and your intended use is not permitted by statutory regulation or exceeds the permitted use, you will need to obtain permission directly from the copyright holder. 